\title{
The effect and potential risks of using cement- augmented pedicle screw instrumentation on multiple segment fixation in osteoporotic spine with lumbar degenerative diseases
}

\section{Yong-chao Tang}

Guangzhou University of Chinese Medicine

Hui-zhi Guo

Guangzhou University of Chinese Medicine

Dan-qing Guo

Guangzhou University of Chinese Medicine

Pei-jie Luo

Guangdong Hospital of Traditional Chinese Medicine

\section{Yong-xian Li}

Guangzhou University of Chinese Medicine

\section{Guo-ye Mo}

Guangzhou University of Chinese Medicine

\section{Yan-huai Ma}

Guangzhou University of Chinese Medicine

Jian-cheng Peng

Guangzhou University of Chinese Medicine

De Liang

Guangzhou University of Chinese Medicine

Shun-cong Zhang (D 18122436960@163.com)

Guangzhou University of Chinese Medicine https://orcid.org/0000-0003-3952-0030

\section{Research article}

Keywords: Multiple segment fixation, Cement-augmented pedicle screw instrumentation, Lumbar degenerative diseases, Selective cement-augmenting

Posted Date: March 3rd, 2020

DOI: https://doi.org/10.21203/rs.2.19693/v2 
License: (c) (i) This work is licensed under a Creative Commons Attribution 4.0 International License. Read Full License

Version of Record: A version of this preprint was published at BMC Musculoskeletal Disorders on April 28th, 2020. See the published version at https://doi.org/10.1186/s12891-020-03309-y. 


\section{Abstract}

Background: Cement-augmented pedicle screw instrumentation (CAPSI) has been found to significantly increase the pedicle screws anchorage in the osteoporotic vertebral body, however, with the increase of augmented level and bone cement dose, the incidence of cement leakage (CL) or side complication of CAPSI also increased. But the effect and potential risks of CAPSI for osteoporotic lumbar degenerative diseases (LDD) have not been investigated in the cases of multiple segment fixation. The purpose of this study was to investigate potential complications of using cement-augmented screws for multilevel fixation (three or four levels) and the effectiveness of this method for treating LDD with low bone quality.

Methods: Our clinical database was reviewed for osteoporotic patients who using CAPSI or conventional pedicle screw (CPS) for multilevel fixation (three or four levels) between February 2010 to February 2017. A total of 93 patients with multilevel LDD were divided into the CAPSI group (46 subjects) and the CPS group (47 subjects), including 75 cases for three levels (L2-L5 21 cases $\square$ L3-S1 54 cases), 18 cases for four levels (L1-L5 2 cases $\square$ L2-S1 16 cases), and the average follow-up was $33.72 \pm 18.78$ months (range: 24-108). Relevant data were recorded, including age, gender, body mass index (BMI), bone mineral density (BMD), diagnosis, fusion methods, bone graft, fusion level, lumbosacral fixation, different patterns of S1 pedicle screw placement, operation time, blood loss, hospital stay, complications, $\mathrm{CL}$, pulmonary cement embolism (PCE), screw loosening and nonunion patients. Besides, the visual analogue scale (VAS) and Oswestry disability index (ODI) at pre-operation, post-operation, and follow-up were also assessed.

Results: In the CAPSI group, a total of 336 augmented screws were placed bilaterally, and the average injection dose of bone cement was $1.85 \pm 0.65 \mathrm{ml}$ (range: 1-4). The CL was observed in 44 patients (95.65\%) and 116 screws (34.52\%). Three cemented screws $(0.89 \%)$ and twenty-four S1 conventional screws (44.44\%) were found loosening during the follow-up and the overall fusion rate was $93.47 \%$. For perioperative complications, two patients (4.35\%) experienced PCE, one patient augmented vertebral fracture and three patients (6.52\%) wound infection. And in the CPS group, seventeen patients (36.17\%) and thirty-three screws (8.46\%) suffered loosening in cranial or caudal segments (seven cranial cases and twenty-six of caudal), four cases experienced nonunion, the fusion rate was $91.49 \%$ in total. The operation time and hospital stay were longer in CAPSI group, but with a lower percentage of screw loosening ( $P \otimes 0.05)$. And in terms of blood loss, perioperative complications, fusion rate, VAS and ODI scores at the follow-up times, no significant differences were found between the two groups.

Conclusions: Patients with osteoporotic LDD underwent CPS at multiple segments have a high rate of screw loosening in the cranial and caudal segment. The application of cemented pedicle screws on multilevel LDD can achieve better stability and less screw loosening, but it also accompanied by longer operating time, higher incidence of $\mathrm{CL}, \mathrm{PCE}$ and wound infections. Selective cement-augmenting the cranial and caudal pedicle screws may be a worthy strategy to decrease the complications.

\section{Background}


Pedicle screw fixation is widely used in degenerative lumbosacral disease, secondary kyphosis or deformity, and thoracolumbar fractures due to a variety of indications, such as bony fusion promotion, deformity correction and fixation for vertebral fractures [1-3]. Although it is the gold standard for the treatment of degenerative and traumatic spinal diseases benefited from its solid fixation [2], it's purchase strength of low-quality bone still reduced significantly in rising osteoporotic patients $[4,5]$. Instrument failure, such as screw loosening and back-out, associated with nonunion, pseudoarthrosis and progressive kyphosis, is the most common complication of posterior internal fixation in osteoporosis patients $[6,7]$.

BMD plays a pivotal role in the stability of the pedicle screw[8], which can be also affected by other factors include age, smoking, diabetes, different spinal diseases, fusion methods, fixation segments, sacral fixation, repeated screw placement during operation, spinopelvic parameters and geometry shape of screw[9-12]. Studies have shown that the rate of screw loosening is $1 \%-15 \%$ in non-osteoporotic patients, but up to $10 \%-60 \%$ in osteoporotic patients $[9,10]$ and even $50 \%-62.8 \%$ in multi-segment fixation [11-13]. Since both osteoporosis and multilevel LDD are the common disorders in elderly patients [2], improving the attachment of internal instruments for patients with multilevel osteoporotic LDD has become a mission for spine surgeons.

Both fenestrated screws and solid screws with cement-augmented has been proved effective to improve the pullout strength of screws and reduce the risk of fixation failure by biomechanical studies and clinical trials $[14,15]$. But CL remains a common complication of CAPSI, and the leakage rate is from $11.6 \%$ to $82.4 \%$ in bone cement-augmented pedicle screw fixation of the thoracolumbar spine $[16,17]$, which may cause severe complications such as nerve injury, vascular damage, pulmonary embolism, cardiac embolism and anaphylactic shock $[17,18]$. Notably, a multilevel cement-augmented pedicle screw in osteoporotic bone is considered to lead to the high incidence of $\mathrm{CL}$ and cement-related complications. But we found that there are no studies selectively analyzed the effect and potential risks of CAPSI for multiple segment fixation in osteoporotic spine with lumbar degenerative diseases (LDD). Therefore, the present study aims to compare the clinical and radiological results of osteoporotic patients with multilevel LDD (three or four levels) who were treated with CAPSI versus those who were treated with CPS.

\section{Methods}

\section{Patient Population}

From February 2010 to February 2017, the patients visiting our hospital for CAPSI or CPS were recruited for the study. The inclusion criteria as follow: (1) diagnosis with the LDD by symptoms, signs, and imaging examinations who were no response to at least 3 months of conservative treatment; (2) patients with posterior interbody fusion and using consecutive 3-level (L2-L5 or L3-S1) or 4-level (L1-L5 or L2-S1) pedicle screw implanting; and (3) with lumbar vertebral bone mineral density (BMD) measured by dualenergy X-ray absorptiometry and T $\leq-2.5 \mathrm{SD}$. The Exclusion criteria as follow: (1) patients with vertebral 
fracture, tumor or infection; (2) patients with lumbar surgery history; (3) patients with partial lumbar screws were augmented in the CAPSI group or (4) incomplete imaging data in follow-up.

Ninety-three patients, including 75 cases for three levels (L2-L5 21 cases[L3-S1 54 cases) and 18 cases

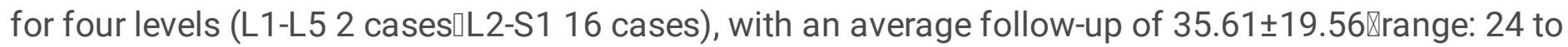
$108 \rrbracket$ months were divided into the CAPSI group (46 subjects) and the CPS group (47 subjects). In the CAPSI group, all patients had received cement-augmented screws at lumbar vertebra, but only eight patients adopted augmented screws and twenty-seven used conventional pedicle screws at S1 because of lacking the technique of sacral pedicle screw with cement augmentation which would be used later. CAPSI group had 7 males and 39 females with a mean age of $70.65 \pm 7.20$ (range: 52 86) years, T scores of $-3.18 \pm 0.94$ (range: $-2.5 \sim-4.9$ ) SD and follow-up of 31.87 \pm 15.49 هrange: $24 \sim 108 \bigotimes$ months. Twenty-five patients had lumbar spinal stenosis with spinal instability, nine had lumbar spondylolisthesis, and twelve had lumbar spinal stenosis with degenerative scoliosis. Solid screws were used in 16 patients and fenestrated screws in 30 patients. CAP group had 6 males and 41 females with a mean age of $67.91 \pm 7.62$ (range: $55 \sim 86$ ) years, T scores of $-2.89 \pm 0.49$ (range: $-2.5 \sim-5.0$ ) SD and follow-up of 35.53 \pm 21.54 『range: $24 \sim 96 \rrbracket$ months. Thirty patients had lumbar spinal stenosis with spinal instability, eight had lumbar spondylolisthesis, and nine had lumbar spinal stenosis with degenerative scoliosis. The flowchart of this study is shown in Figure 1.

\section{Surgical technique}

All patients were performed standard posterior lumbar interbody fusion (PLIF) or (and) transforaminal lumbar interbody fusion (TLIF) under general anesthesia. The decision for cement augmentation was made by an experienced surgeon after evaluate patients' BMD and the biomechanical stability of the implanted pedicle screw. The operation of traditional pedicle screw was using a standard technique according to the literature $[4,10]$. The procedure of fenestrated pedicle screw with cement and solid pedicle screw with pre-augmented trajectory were described in our previous study[19]. In general, pedicle screws with a diameter and a length of $6.5 \mathrm{~mm}$ and $45 \mathrm{~mm}$ were used in lumbar, and a diameter and a length of $6.5 \mathrm{~mm}$ and $40 \mathrm{~mm}$ was used in the sacrum. Besides, the polymethylmethacrylate (PMMA) was injected with approximately 1.5 to $4 \mathrm{~mL}$ for every lumbar pedicle screw and $1.5-2.5 \mathrm{ml}$ for every sacral pedicle screw.

At early stage after operation, the patients were required to perform both lower limbs exercises on the bed. The drainage tube was removed when the amount of drainage was less than $50 \mathrm{ml} / \mathrm{d}$. All patients began to get out of bed and walk with the protection of waist circumference three or four days after the operation. Patients were required to wear a waist circumference withing first month after operation. Routine postoperative X-ray of the thorax were applied in augmented patients, and patients complain of discomfort in the heart or lung will receive additional thoracic CT. All patients received anti-osteoporosis treatment through the whole period of treatment: calcium carbonate, vitamin D3, and bisphosphonate. 


\section{Evaluation indicators}

The Visual Analog Scale (VAS) and the Oswestry Disability Index (ODI) at different follow-up periods were recorded to assess the clinical outcomes. Procedure duration, intraoperative blood loss, and duration of hospitalization of two groups were abstracted from medical records. Major postoperative complications were also compared, including nerve injury, dural tearing, wound infection, and revision surgery.

Radiologic evaluation included spinopelvic parameters, screw loosening rate, and lumbar fusion status. Two experienced orthopedists were assigned to evaluate the radiologic parameters independently. All the data were measured twice by two researchers, and the mean values were used for analysis. The degree of lumbar lordosis (LL), pelvic title (PT) and pelvic incidence (PI) were assessed according to the previous studies [20]. Screw loosening was defined as a halo sign showing a radiolucent line of $\geq 1 \mathrm{~mm}$ around the screw in X-rays or CT images in one or both sides after the surgery [11, 21]. Solid fusion was determined according to the criterion described by Dakhil et al [22], as a bridging interbody bone occurs in the operated segment and an angle of less than $5^{\circ}$ on dynamic x-ray images in the fixed segments.

\section{Statistical Method}

The data were analyzed using the SPSS 19.0 software (IBM, Inc., Armonk, NY, USA). The difference of the continuous variables between the two groups were compared by using the independent sample $t$-test (age, BMD, BMI, VAS and ODI scores, follow-up, operation time, blood loss, and hospital stay and spinopelvic parameters). The chi-square test was used to compare the classification variables (gender, diabetes, hypertension, smoking, diagnosis, fusion methods, bone graft, fusion level, lumbosacral fixation, different patterns of S1 pedicle screw placement, lumbosacral fixation, complications, screw loosening, nonunion patients). A $P<0.05$ was considered statistically significant.

\section{Results}

\section{Baseline characteristics}

There were no statistical differences among these two groups in term of gender, age, BMD, BMI, diabetes, hypertension, smoking, diagnosis, fusion methods, bone graft, fusion level, lumbosacral fixation, different patterns of $\mathrm{S} 1$ pedicle screw placement, lumbosacral fixation, preoperative VAS score, preoperative ODI score, follow-up, pre-operation LL, pre-operation PT, and pre-operation PI (Table 1).

\section{Clinical effects and complications}


336 pedicle trajectories were cement-augmented, with a mean of $7.30 \pm 1.4$ (range: $6 \sim 10$ ) instrumented screw and a mean of $1.85 \pm 0.65$ (range:1 4) ml PMMA per pedicle screw was used. The CL was observed in 44 patients (95.65\%) and 116 screws (34.52\%), and CL most often occurred in the perivertebral venous system: leakage via segmental veins and basivertebral veins were seen in 109 of screws $(93.97 \%)$. Perioperative complications included two patients (4.35\%) had PCE (one was symptomatic and the other was asymptomatic) (Fig. 2), two patients had hypotension, two patients had a superficial skin infection that required surgical debridement, one patient had deep infection but the instrumentation was not removed, one patient had dural tear and were discharged after surgical suture repair, one patient had augmented vertebral fracture (Fig. 3), six patients experienced a leg radiating pain and two patients still complain about discomfort at the last follow-up. There were no serious complications caused by the leakage of bone cement, such as nerve damage, cardiac embolism or death. Three cemented screws (0.89\%) (Fig. 4) and twenty-four S1 conventional screws (44.44\%) were found loosening at the follow-up, three patients have nonunion and two of them required revision surgery with a fusion rate of $93.47 \%$. Adjacent fractures were observed in six patients and eight vertebral during follow up, symptomatic adjacent disc degeneration was observed in 3 patients and two of them required revision surgery. $A$ typical case of CAPSI group is shown in Figure 5.

In the CPS group, perioperative complications included one patient had hypotension, one patient had a dural tear, and eight patients experienced severe leg radiating pain due to the pedicle screws misplacement (three of them still complain about discomfort at the last follow-up). Seventeen patients \36.17\% \and thirty-three screws(8.46\%囚showed loosening in the cranial or caudal segment (Fig. 6), four cases experienced nonunion and one of them required revision surgery with a fusion rate of $91.49 \%$. Adjacent fractures were observed in three patients and three vertebral during follow up, symptomatic adjacent disc degeneration was observed in three patients and all of them required revision surgery. A typical case of CPS group is shown in Figure 7.

Compared with the date of pre-operation, larger post-operation LL were found in both CAPSI group and CPS group. But there were no statistical differences in PT and PI between pre-operation and postoperation in each group. Longer operation time, longer hospital stay and less screw loosening were found in the CAPSI group (Pख0.05). And in terms of blood loss, perioperative complications, fusion rate, VAS and ODI scores at the follow-up, post-operation LL, post-operation PT, and post-operation PI, no significant difference detected between the two groups (Table 2 and Figure 8).

\section{Discussion}

Pedicle screw fixation in osteoporotic patients is gradually becoming common because of the aging of the population. Therefore, an increasing incidence of pedicle screw loosening and instrumentationassociated complications will inevitably be faced [23]. According to the literature, $17 \%$ of revision surgeries are associated with pedicle screw failure [5]. Thus, CAPSI has received growing attention due to its reliable fixation and simple surgical technique, which has been shown to increase pedicle screw pullout force from $147 \%$ to $278 \%$ in both vitro and in vivo clinical studies $[15,24-26]$. But the treatment of 
multilevel LDD with cement-augmented pedicle screws is more complicated and challenging compared with 1-level or 2-level LDD for the increase of CL and mechanical overload [27]. Many studies either assessed only the results of short segment augmentation or assessed the results of long and short segment augmentation together, and most data do not relate to osteoporotic LDD. There is currently little data on the effectiveness and potential risks of CAPSI when treating LDD affecting 3 or more levels.

Although the application of cemented pedicle screw on multilevel LDD can achieve better stability with less screw loosening, it's also bears a high risk of $C L, P C E$, and wound infections. The $C L$ rate occurring in the present study was $34.52 \%$ and $93.97 \% \mathrm{CL}$ was leakage into segmental veins and basivertebral veins. Several studies indicated that CAPSI bears a very high risk of asymptomatic cement leakage, of which a considerable fraction becomes clinically symptomatic. Methods to reduce CL in LDD include: (1) Using high viscosity cement or toothpaste viscosity of cement[28] (the waiting period after mixing powder and liquid was 390 seconds in this study). (2) Reducing the volume of cement and the number of augmented screws. The large volume of bone cement and augmented screw resulting in increasing possibility of secondary venous wall rupture and cement pressed in $[29,30]$. Experimental studies also have indicated that the screw stability doesn't significantly improve with the volumes of PMMA above $2.8 \mathrm{ml}$ each screw [25]. Therefore, the volumes of injected PMMA should be optimized according to the different osteoporotic spine and should $1.5-2.5 \mathrm{ml}$ per pedicle screw. (3) Planning the angle of screw placement and the selection of screw length by preoperative CT careful, so that surgeons can reduce the incidence of screw penetrating the bone cortex and the tip of screw approaching to the midline of the vertebral body. Our previous study has found that a smaller distance between the tip of the screw and the midline was a significant risk factor for epidural $\mathrm{CL}[19]$. (4) We propose injecting small doses slowly (the cement was injected by every $0.1 \mathrm{ml}$ increment in this study) or creating a small cavity in the vertebral body prior to cement injection [29]. (5) The injection of cement should be confirmed consecutively by fluoroscopy.

In this study, PCE was detected in two cases (4.35\%), including one patient was symptomatic. Researchers have shown the risk of pulmonary embolism after percutaneous vertebroplasty and kyphoplasty ranging from $3.5 \%$ to $23 \%$. This variation is relate to the radiographic technique used to evaluate embolism: CT images are more sensitive in detect small embolism [17, 31]. Most of the pulmonary embolism is asymptomatic and the incidence of symptomatic pulmonary embolism is about $1.4 \% \sim 4.1 \%$ [31]. The happening of deaths is scarce and related to fulminant or severe PCE [17, 32]. The optimal treatment strategy for PCE remains controversial. Many authors have suggested that in case of asymptomatic PCE, clinical observation and regular follow-up without anticoagulants is feasible. For patients with symptomatic PCE, long-term anticoagulation therapy can improve the safety of patients, included initial heparin during hospitalization and oral coumarin therapy at the 6-month follow-up. In severe symptomatic cases, surgical intervention to remove cement embolus is also reported in some patients[17].

A wound infection could be a risk factor for a concomitant vertebral body infection and instrumentation removal. The rate of infection in CAPSI group was very high (6.52\%), we analyzed our data to explain the high infection rate, and we found that the infect patients with average age of 78 years old, average 
operation time of $326.67 \mathrm{~min}$, average blood loss of $1433.33 \mathrm{ml}$, and two of them have diabetes. We believe that the high infection rate was related to the elderly age, long operation time, a large amount of bleeding and other comorbidities, such as diabetes. We are very aggressive in the management of possible deep infection. Whenever it is suspected, rapid and aggressive surgical debridement is performed. Martín-Fernández [16] also think it is necessary to control infection early and rapidly, in his study, all surgical incision was performed to rapid and aggressive surgical debridement and to antibiotic treatment. Interbody and posterior segmental instrumentation can usually be left in place early on, as several authors have reported high success rates using this hardware preservation strategy in the management of early surgical site.

Few clinical studies reported the loosening of the augmented screw, but which can't mask the fact that it is one of the complications in CAPSI. Two patients and three augmented screws were found loosening in our study, one of which is caused by tumble and the others are caused by poor distribution of bone cement around the screws. Individuals with LDD always have sensory, proprioception and musculoskeletal system alterations, which may result in balance impairment. Although these people's balance control, pain, and functional activities were observed improved after lumbar surgery, they were still less stable than those age-matched healthy adults after surgery[33]. Therefore, these LDD patients are more likely to fall, especially those who underwent multilevel fixation. When bone cement was poorlydispersed around the pedicle screw, the augmented bone intensity, pull-out strength, periodic loading times and compression-fatigue performance were markedly lower than those well distributed one[14, 23]. In addition, according to our experience, when solid screw with PMMA augmentation was implanted late and cement become solidified, the sclerotic wall of the cement did not allow the screw to interdigitate into the PMMA, and thus the screw may deviate from augmented trajectory while the cement-screw interface was poorly-dispersed. This is another factor of augmented screw loosening.

We attempted to inject PMMA and then place the screws immediately on multilevel augmentation from the beginning, but it was with the risk that screw cannot interdigitate into sclerotic cement (Fig.3). Later we found using fenestrated screws allows for better control of PMMA injection (especially more than two levels need to be instrumented), and augment of the levels based on both the BMD and primary attachment of the screw. Thus, we propose using fenestrated screws on multilevel augmentation, especially for inexperienced surgeons. But we tend to insert the solid screws instead of fenestrated screws in $\mathrm{S} 1$ to reduce the leakage caused by fenestrated screws penetrating the presacral bone cortex [20]. Otherwise, we usually use augmented screws on two vertebral bodies at a time rather than three bodies, minimizing the difficulty of operation and facilitating C-arm fluoroscopy.

Screw loosening of multi-segment fixation often occurs in the cranial and caudal segment, much more frequently in the caudal (S1 in particular). Coincided with previous studies [9, 27], twenty-four S1 conventional screws(44.44\%) were found loosening in the CAPSI group, and thirty-three screws(8.46\%) in CPS group, including seven screws in cranial and twenty-six caudal (eight in L5, eighteen in S1). Wu et al[9] analyzed 658 screws in 126 patients and found that 25 patients experienced screw loosening, 18 patients $(72 \%)$ had loosened screws in the inferior portion of the spinal construct, while $7(28 \%)$ in the 
superior portion. Li et al[27] also found that instrument failure (broken screw or rod) usually develops at the lowest segment in cases of multilevel pedicle screw fixation. The cause of this trend may be the mechanical overload on the cranial and caudal, which results in stress concentration on the cranial or caudal pedicle screw. Moreover, S1 pedicles have a larger diameter and shorter length than the lumbar ones, and the bone-screw interface located in the weaker trabecular bone of the sacrum may also contribute to the loosing of S1 screws. Therefore, we recommend that it is necessary for properly increasing bone cement dose in the cranial and caudal screw and the augment of S1 screw because these methods can reduce the need for sacroiliac screw fixation and other pelvic fixation [20].

Since the application of pedicle screws with cement on all segments will increase the operating time, $\mathrm{CL}$, PCE and incision infection, only cementing the selected strategic vertebrae, such as the cranial and caudal pedicle screw alone, could be a worthy strategy of choice. Based on the results of clinical and radiological data compared 31 patients, with degenerative spinal pathologies, who experienced treatment of pedicle screws and cement injections on all segments to those who treated with cement injections only on the strategic vertebrae selected, Erdem[34] argued that the cement embolism, symptomatic chest discomfort, and duration of surgery were significantly higher in all segments cement injections. Therefore, cementing the strategic vertebrae alone can enhance the fixation strength and endurance as well as decrease the side complications of cement application.

There are limitations of our study, since we have not analyzed the potential impacts of the screw loosening and fusion, such as the strength of paravertebral muscles, single or double cage, the length/diameter of screw and the repeated placement of screws during operation, as well as for the specific time of screw loosening and asymptomatic adjacent segment degeneration. In addition, the actual rate of loosening may be lower than those reported in this paper, because the patients with screw loosening are more likely to receive follow-up, while patients with good postoperative recovery may be reluctant to return to the hospital.

\section{Conclusions}

Patients with osteoporotic LDD underwent CPS at multiple segments have a high rate of screw loosening in the cranial and caudal segment. The application of cemented pedicle screws on multilevel LDD can achieve better stability and less screw loosening, but it also accompanied by longer operating time, higher incidence of CL, PCE and wound infections. Selective cement-augmenting the cranial and caudal pedicle screws may be a worthy strategy to decrease the complications.

\section{Abbreviations}

CAPSI: Cement-augmented pedicle screw instrumentation; CL: cement leakage; LDD: lumbar degenerative diseases; CPS: conventional pedicle screw; PCE: pulmonary cement embolism ; BMD: bone mineral density ; BMI: Body Mass Index; VAS: Visual Analogue Scale; ODI: Oswestry Disability Index; PMMA: 
polymethylmethacrylate ; CT: Computed tomography; TLIF: transforaminal lumbar interbody fusion ;

PEEK: polyether ether ketone; LL: lumbar lordosis ; PT: Pelvic title; PI: Pelvic incidence

\section{Declarations}

\section{Ethics approval and consent to participate}

This study was approved by the Ethics Committee of The First Affiliated Hospital of Guangzhou University of Chinese Medicine. All participation in the study was voluntary and written informed consent was obtained from the participants.

\section{Consent for publication}

Not applicable.

\section{Availability of data and materials}

The datasets used in the current study are not publicly available due to privacy protection but are available from the corresponding author on reasonable request.

\section{Competing interests}

The authors declare that they have no competing interests.

\section{Funding}

This study supported by the innovation and strength project of The First Affiliated Hospital of Guangzhou University of Chinese Medicine (2019هT32). The funders had no role in study design, data collection or analysis, decision to publish, or preparation of the manuscript.

\section{Authors' contributions}

YT and HG conceived the study, designed the study, drafted the manuscript and had the overall scientific responsibility. $\mathrm{DG}, \mathrm{PL}$, and $\mathrm{YL}$ contributed to the study design, data analysis and interpretation of data. $\mathrm{GM}, \mathrm{YM}, \mathrm{JP}$, and $\mathrm{DL}$ contributed to the data collection, interpretation of data and paper revising. All authors contributed to the paper revision, read and approved the final manuscript. 


\section{Acknowledgements}

None.

\section{Authors' details}

${ }^{1}$ The First Clinical Academy, Guangzhou University of Chinese Medicine, Guangzhou, China. ${ }^{2}$ Spine Surgery, The First Affiliated Hospital of Guangzhou University of Chinese Medicine, Guangzhou, China

\section{References}

1. Moussazadeh N, Rubin DG, McLaughlin L, Lis E, Bilsky MH, Laufer I. Short-segment percutaneous pedicle screw fixation with cement augmentation for tumor-induced spinal instability. 2015. 2015;15(7):1609-17.

2. Rihn JA, Hilibrand AS, Zhao W, et al. Effectiveness of surgery for lumbar stenosis and degenerative spondylolisthesis in the octogenarian population: analysis of the Spine Patient Outcomes Research Trial (SPORT) data. 2015. 2015;97(3):177-85.

3. Rong Z, Zhang F, Xiao J, et al. Application of Cement-Injectable Cannulated Pedicle Screw in Treatment of Osteoporotic Thoracolumbar Vertebral Compression Fracture (AO Type A): A Retrospective Study of 28 Cases. 2018. 2018;120:e247-247e258.

4. Weiser L, Huber G, Sellenschloh K, et al. Insufficient stability of pedicle screws in osteoporotic vertebrae: biomechanical correlation of bone mineral density and pedicle screw fixation strength. 2017. 2017;26(11):2891-7.

5. Bostelmann R, Keiler A, Steiger HJ, Scholz A, Cornelius JF, Schmoelz W. Erratum to: Effect of augmentation techniques on the failure of pedicle screws under cranio-caudal cyclic loading. 2015. 2015;24(9):2098.

6. Weiser L, Dreimann M, Huber G, et al. Cement augmentation versus extended dorsal instrumentation in the treatment of osteoporotic vertebral fractures: a biomechanical comparison. 2016. 2016;98B(8):1099-105.

7. Park CK, Park CK, Lee DC, Lee DG. A new technique of bone cement augmentation via the disc space for percutaneous pedicle screw fixation. 2016. 2016;24(1):16-9.

8. Oberkircher L, Krüger A, Hörth $D$, et al. Anterior cement augmentation of adjacent levels after vertebral body replacement leads to superior stability of the corpectomy cage under cyclic loading-a biomechanical investigation. 2018. 2018;18(3):525-31.

9. Wu JC, Huang WC, Tsai HW, et al. Pedicle screw loosening in dynamic stabilization: incidence, risk, and outcome in 126 patients. 2011. 2011;31(4):E9. 
10. Galbusera F, Volkheimer D, Reitmaier S, Berger-Roscher N, Kienle A, Wilke HJ. Pedicle screw loosening: a clinically relevant complication. 2015. 2015;24(5):1005-16.

11. Kim JB, Park SW, Lee YS, Nam TK, Park YS, Kim YB. The Effects of Spinopelvic Parameters and Paraspinal Muscle Degeneration on S1 Screw Loosening. 2015. 2015;58(4):357-62.

12. Röllinghoff M, Schlüter-Brust K, Groos D, et al. Mid-range outcomes in 64 consecutive cases of multilevel fusion for degenerative diseases of the lumbar spine. 2010. 2010;2(1):e3.

13. El Saman A, Meier S, Sander A, Kelm A, Marzi I, Laurer H. Reduced loosening rate and loss of correction following posterior stabilization with or without PMMA augmentation of pedicle screws in vertebral fractures in the elderly. 2013. 2013;39(5):455-60.

14. Choma TJ, Frevert WF, Carson WL, Waters NP, Pfeiffer FM. Biomechanical analysis of pedicle screws in osteoporotic bone with bioactive cement augmentation using simulated in vivo multicomponent loading. 2011. 2011;36(6):454-62.

15. Tan JS, Bailey CS, Dvorak MF, Fisher CG, Cripton PA, Oxland TR. Cement augmentation of vertebral screws enhances the interface strength between interbody device and vertebral body. 2007. 2007;32(3):334-41.

16. Martín-Fernández M, López-Herradón A, Piñera AR, et al. Potential risks of using cement-augmented screws for spinal fusion in patients with low bone quality. 2017. 2017;17(8):1192-9.

17. Janssen I, Ryang YM, Gempt J, et al. Risk of cement leakage and pulmonary embolism by bone cement-augmented pedicle screw fixation of the thoracolumbar spine. 2017. 2017;17(6):837-44.

18. Hatzantonis C, Czyz M, Pyzik R, Boszczyk BM. Intracardiac bone cement embolism as a complication of vertebroplasty: management strategy. 2017. 2017;26(12):3199-205.

19. Guo HZ, Tang YC, Guo DQ, et al. The cement leakage in cement-augmented pedicle screw instrumentation in degenerative lumbosacral diseases: a retrospective analysis of 202 cases and 950 augmented pedicle screws. 2019. 2019.

20. Guo HZ, Tang YC, Li YX, et al. The Effect and Safety of Polymethylmethacrylate-Augmented Sacral Pedicle Screws Applied in Osteoporotic Spine with Lumbosacral Degenerative Disease: A 2-Year Follow-up of 25 Patients. 2019. 2019;121:e404-404e410.

21. Moon BJ, Cho BY, Choi EY, Zhang HY. Polymethylmethacrylate-augmented screw fixation for stabilization of the osteoporotic spine : a three-year follow-up of 37 patients. 2009. 2009;46(4):30511.

22. Dakhil-Jerew F, Jadeja H, Cohen A, Shepperd JA. Inter-observer reliability of detecting Dynesys pedicle screw using plain X-rays: a study on 50 post-operative patients. 2009. 2009;18(10):1486-93.

23. Fan HT, Zhang RJ, Shen CL, et al. The Biomechanical Properties of Pedicle Screw Fixation Combined With Trajectory Bone Cement Augmentation in Osteoporotic Vertebrae. 2016. 2016;29(2):78-85.

24. Bullmann V, Liljenqvist UR, Rödl R, Schulte TL. [Pedicle screw augmentation from a biomechanical perspective]. 2010. 2010;39(7):673-8. 
25. Fölsch C, Goost H, Figiel J, Paletta JR, Schultz W, Lakemeier S. Correlation of pull-out strength of cement-augmented pedicle screws with CT-volumetric measurement of cement. 2012. 2012;57(6):473-80.

26. Elder BD, Lo SF, Holmes C, et al. The biomechanics of pedicle screw augmentation with cement. 2015. 2015;15(6):1432-45.

27. Li T, Shi L, Luo Y, Chen D, Chen Y. One-Level or Multilevel Interbody Fusion for Multilevel Lumbar Degenerative Diseases: A Prospective Randomized Control Study with a 4-Year Follow-Up. 2018. 2018;110:e815-815e822.

28. Schmoelz W, Heinrichs $\mathrm{CH}$, Schmidt S, et al. Timing of PMMA cement application for pedicle screw augmentation affects screw anchorage. 2017. 2017;26(11):2883-90.

29. Arabmotlagh M, Rickert M, Lukas A, Rauschmann M, Fleege C. Small cavity creation in the vertebral body reduces the rate of cement leakage during vertebroplasty. 2017. 2017;35(1):154-9.

30. Ulusoy OL, Kahraman S, Karalok I, et al. Pulmonary cement embolism following cement-augmented fenestrated pedicle screw fixation in adult spinal deformity patients with severe osteoporosis (analysis of 2978 fenestrated screws). 2018. 2018;27(9):2348-56.

31. Krueger A, Bliemel C, Zettl R, Ruchholtz S. Management of pulmonary cement embolism after percutaneous vertebroplasty and kyphoplasty: a systematic review of the literature. 2009. 2009;18(9):1257-65.

32. Mueller JU, Baldauf J, Marx S, Kirsch M, Schroeder HW, Pillich DT. Cement leakage in pedicle screw augmentation: a prospective analysis of 98 patients and 474 augmented pedicle screws. 2016. 2016;25(1):103-9.

33. Wong WJ, Lai DM, Wang SF, Wang JL, Hsu WL. Changes of balance control in individuals with lumbar degenerative spine disease after lumbar surgery: a longitudinal study. 2019. 2019.

34. Erdem MN, Karaca S, Sarı S, Yumrukcal F, Tanli R, Aydogan M. Application of cement on strategic vertebrae in the treatment of the osteoporotic spine. 2017. 2017;17(3):328-37.

\section{Tables}

Table 1 Comparison of baseline data in two groups 


\begin{tabular}{|c|c|c|c|c|}
\hline Factors & CAPSI group $(n=46 \square$ & CPS group $(n=47)$ & & $P$ value \\
\hline Male/female $\llbracket n \square$ & $7 / 39$ & $6 / 41$ & $x^{2}=0.116$ & 0.733 \\
\hline Age (year) & $70.65 \pm 7.20$ & $67.91 \pm 7.62$ & $\mathrm{t}=1.780$ & 0.078 \\
\hline Body mass index & $23.91 \pm 3.36$ & $23.64 \pm 2.85$ & $\mathrm{t}=0.411$ & 0.682 \\
\hline Bone mineral density & $-3.18 \pm 0.94$ & $-2.89 \pm 0.49$ & $\mathrm{t}=-1.822$ & 0.072 \\
\hline Diabetes $\llbracket n \llbracket$ & 12 & 9 & $x^{2}=1.033$ & 0.333 \\
\hline Hypertension $\square n \square$ & 23 & 22 & $x^{2}=0.095$ & 0.837 \\
\hline Smoking $\square \mathrm{n} \square$ & 3 & 2 & $x^{2}=0.284$ & 0.671 \\
\hline Diagnosis (n) & & & $x^{2}=0.933$ & 0.653 \\
\hline Lumbar spinal stenosis & 25 & 30 & & \\
\hline Spondylolisthesis & 9 & 8 & & \\
\hline Degenerative scoliosis & 12 & 9 & & \\
\hline Fusion methods $\llbracket n \square$ & & & $x^{2}=0.732$ & 0.665 \\
\hline TLIF & 26 & 28 & & \\
\hline PLIF & 5 & 7 & & \\
\hline PLIF+TLIF & 15 & 12 & & \\
\hline Bone graft & & & $x^{2}=0.665$ & 0.496 \\
\hline Autograft bone & 15 & 12 & & \\
\hline Autograft and allograft bones & 31 & 35 & & \\
\hline Fusion level $\square n \square$ & & & $x^{2}=3.517$ & 0.522 \\
\hline L2-L5 & 9 & 12 & & \\
\hline L3-S1 & 26 & 28 & & \\
\hline L1-L5 & 2 & 0 & & \\
\hline L2-S1 & 9 & 7 & & \\
\hline Lumbosacral fixation $\square \mathrm{n} \square$ & 35 & 35 & $x^{2}=0.033$ & 1.000 \\
\hline S1 bicortical/tricortical fixation $\square n \square$ & $14 / 21$ & $19 / 16$ & $x^{2}=1.438$ & 0.338 \\
\hline VAS score & $7.19 \pm 0.86$ & $7.34 \pm 0.73$ & $t=-0.876$ & 0.383 \\
\hline ODI score $\llbracket \square$ & $52.04 \pm 7.58$ & $51.11 \pm 7.46$ & $\mathrm{t}=0.601$ & 0.549 \\
\hline Follow-up time $\llbracket m o n t h s \rrbracket$ & $31.87 \pm 15.49$ & $35.53 \pm 21.54$ & $\mathrm{t}=-0.94$ & 0.350 \\
\hline Pre-operation $L L \square^{\circ} \square$ & $31.19 \pm 18.27$ & $35.68 \pm 13.53$ & $t=-1.350$ & 0.180 \\
\hline Pre-operation $\mathrm{PT}^{\circ} \mathrm{\square}$ & $23.50 \pm 10.49$ & $19.76 \pm 8.96$ & $\mathrm{t}=1.850$ & 0.068 \\
\hline Pre-operation $\mathrm{PI}^{\circ}{ }^{\circ}$ & $48.07 \pm 12.61$ & $51.30 \pm 13.54$ & $t=-1.189$ & 0.237 \\
\hline
\end{tabular}

CAPSI Cement-augmented pedicle screw instrumentation, CPS conventional pedicle screw, TLIF transforaminal lumbar interbody fusion, PLIF posterior lumbar interbody fusion, VAS Visual Analogue Scale, ODI Oswestry Disability Index, LL lumbar lordosis, PT Pelvic title, PI Pelvic incidence 
Table 2 Comparison of the operation and clinical effects of two groups

\begin{tabular}{|c|c|c|c|c|}
\hline Factors & CAPSI group $\square n=46 \square$ & CPS group $\square n=47 \square$ & & $P$ value \\
\hline Operation time $\llbracket \min \llbracket$ & $303.07 \pm 61.25$ & $268.72 \pm 64.46$ & $t=2.633$ & 0.010 \\
\hline Blood loss $\square \mathrm{ml} \square$ & $1176.09 \pm 763.82$ & $1072.34 \pm 838.46$ & $\mathrm{t}=0.485$ & 0.629 \\
\hline Hospital stay $\llbracket$ days $\square$ & $24.54 \pm 8.82$ & $20.85 \pm 8.78$ & $t=2.023$ & 0.046 \\
\hline Complications (n $\square$ & 15 & 10 & $x^{2}=1.526$ & 0.249 \\
\hline Post-operation $\mathrm{LL}^{\circ} \square^{\circ}$ & $39.59 \pm 10.55 *$ & $41.57 \pm 9.27 *$ & $\mathrm{t}=-0.958$ & 0.341 \\
\hline Post-operation $\mathrm{PT}^{\circ} \mathrm{a}$ & $21 . .47 \pm 10.18$ & $17.92 \pm 10.14$ & $\mathrm{t}=1.684$ & 0.096 \\
\hline Post-operation $\mathrm{PI}^{\circ}{ }^{\circ} \square$ & $49.47 \pm 12.59$ & $52.03 \pm 12.81$ & $\mathrm{t}=-0.984$ & 0.327 \\
\hline Screw loosening $\square \mathrm{n} \square$ & $3 / 336$ & $33 / 390$ & $x^{2}=23.996$ & 0.000 \\
\hline Nonunion patients $\llbracket \mathrm{n} \square$ & 3 & 4 & $x^{2}=0.133$ & 1.000 \\
\hline Post-operation VAS & $4.19 \pm 1.13^{*}$ & $4.02 \pm 1.39 *$ & $\mathrm{t}=1.973$ & 0.052 \\
\hline Post-operation ODI & $39.30 \pm 7.11 *$ & $36.29 \pm 7.58^{*}$ & $t=0.663$ & 0.509 \\
\hline
\end{tabular}

* Significantly greater than the value found in preoperation

CAPSI Cement-augmented pedicle screw instrumentation, CPS conventional pedicle screw, LL lumbar lordosis, PT Pelvic title, PI Pelvic incidence, VAS Visual Analogue Scale, ODI Oswestry Disability Index.

\section{Figures}




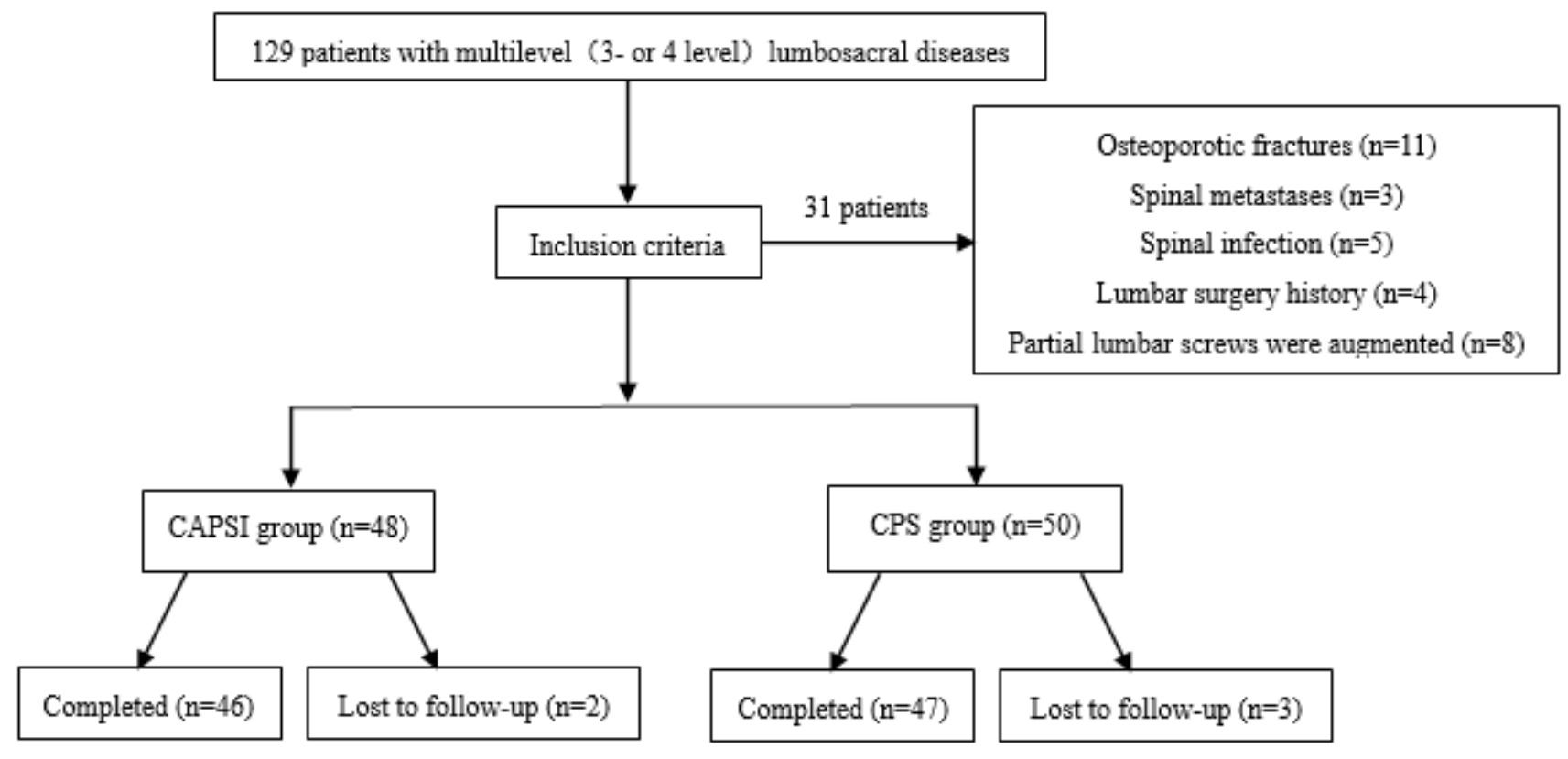

\section{Figure 1}

Diagram showing the process of patient selection.

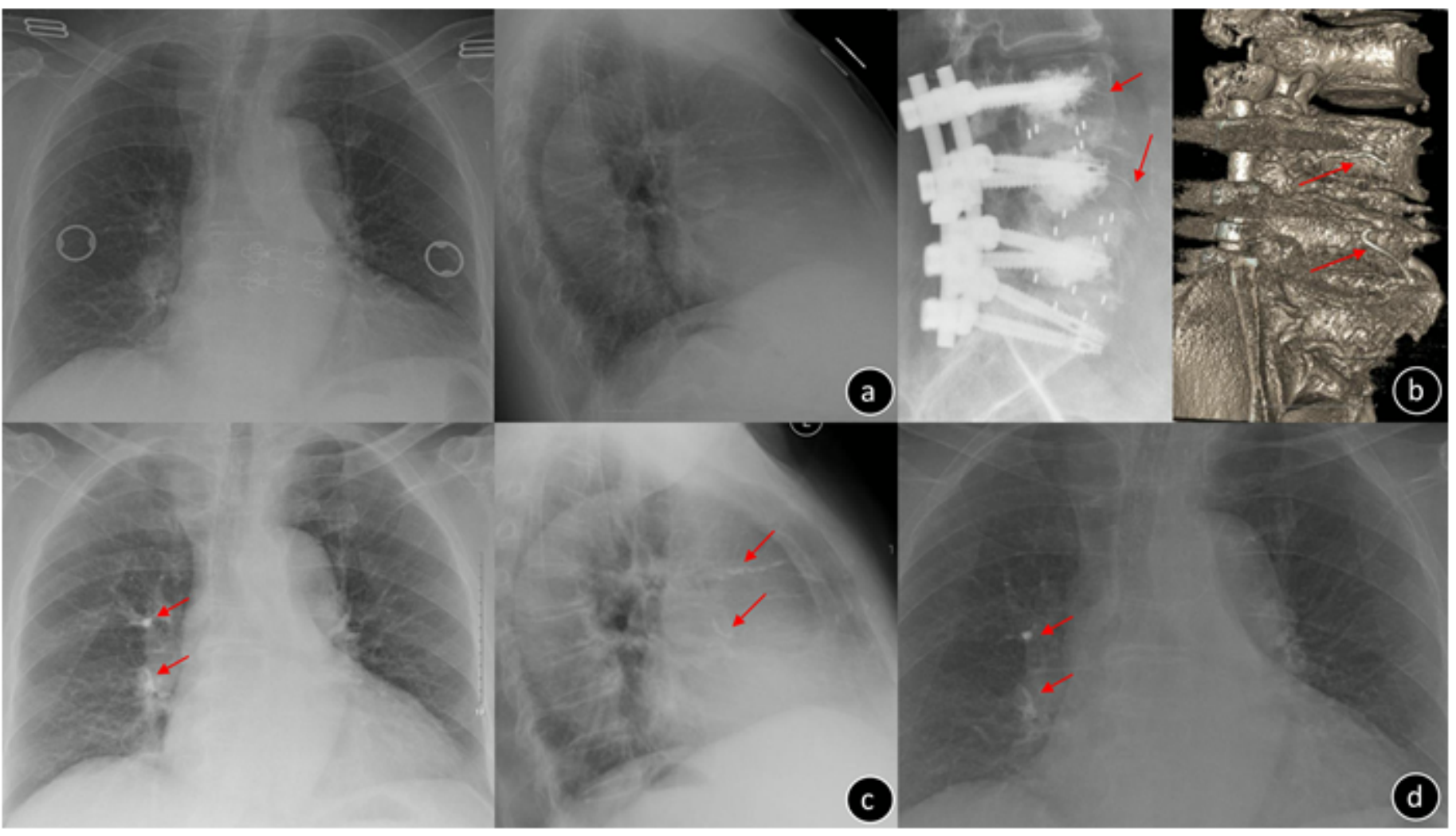

\section{Figure 2}

Female, 73-year-old, T=-3.5 SD a. Preoperative chest x-ray image b. Postoperative x-ray and CT scans illustrating $\mathrm{CL}$ to the segmental vein in L3/L4 (arrow) c. The postoperative anteroposterior radiograph 
showed pulmonary cement embolism on the right side (arrow); this patient experienced post-op dyspnea and blood oxygen desaturation and recovered within six days after oxygen inhalation and anticoagulation treatment $d$. two years after the operation, the chest x-ray showed cement embolism was in situ (arrow) and the patient was asymptomatic.

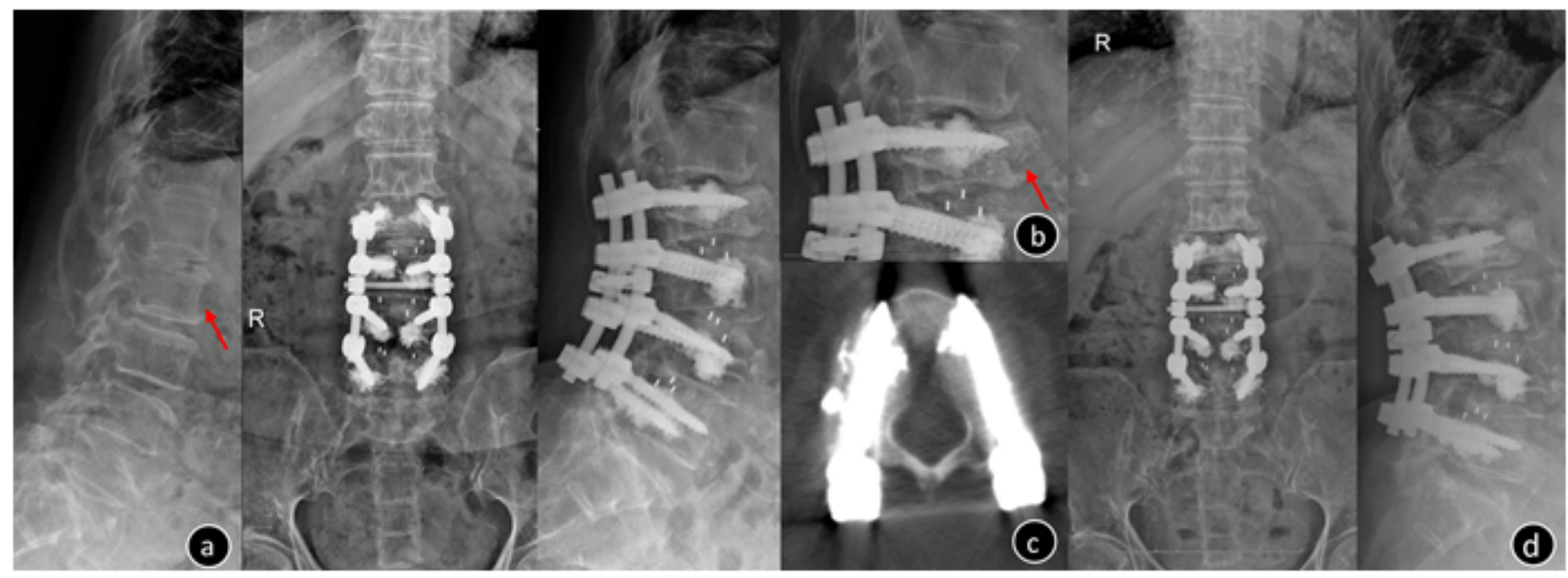

\section{Figure 3}

Female, 73-year-old, $\mathrm{T}=-3.5 \mathrm{SD}$, the patient underwent solid pedicle screw with a pre-augmented trajectory a. The preoperative $x$-ray image showed lumbar vertebral degeneration and no fracture was found in the L2 vertebral; b. Postoperative x-ray: L2 vertebral fracture; $c$ : when a screw was implanted late and the cement becomes solidified, the screw may deviate augmented trajectory, and the following rupture of the cortex bone may result in vertebral fracture; $d$. Three years after the operation, the x-ray showed that L2 vertebral body collapsed, the fracture healed and the L2 pedicle screw has not loosened. 


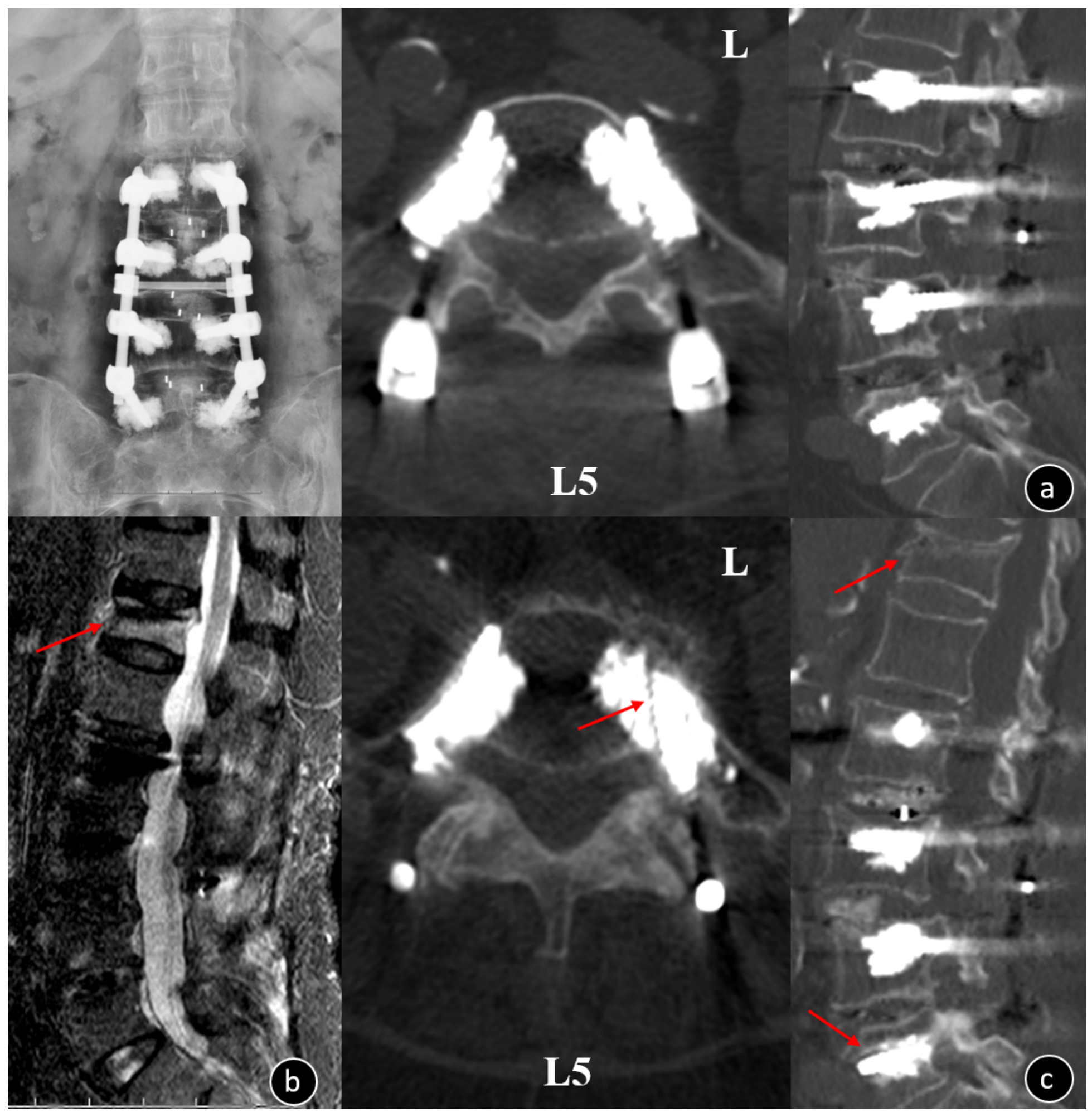

Figure 4

Female, 69-year-old, T=-3.4 SD a. the post-operation X-rays and CT showed the internal fixation was in good position and there was no screw loosening in L5 b. 7 months after the operation, the patient accidentally fell, MRI showed T12 acute osteoporotic vertebral compression fracture; c. the CT illustrated the pedicle screw was loosened in left L5 (arrow). The reason of screw loosening may be related to long segment fixation and stress concentration on the tail of the fixed segment when tumbled. 


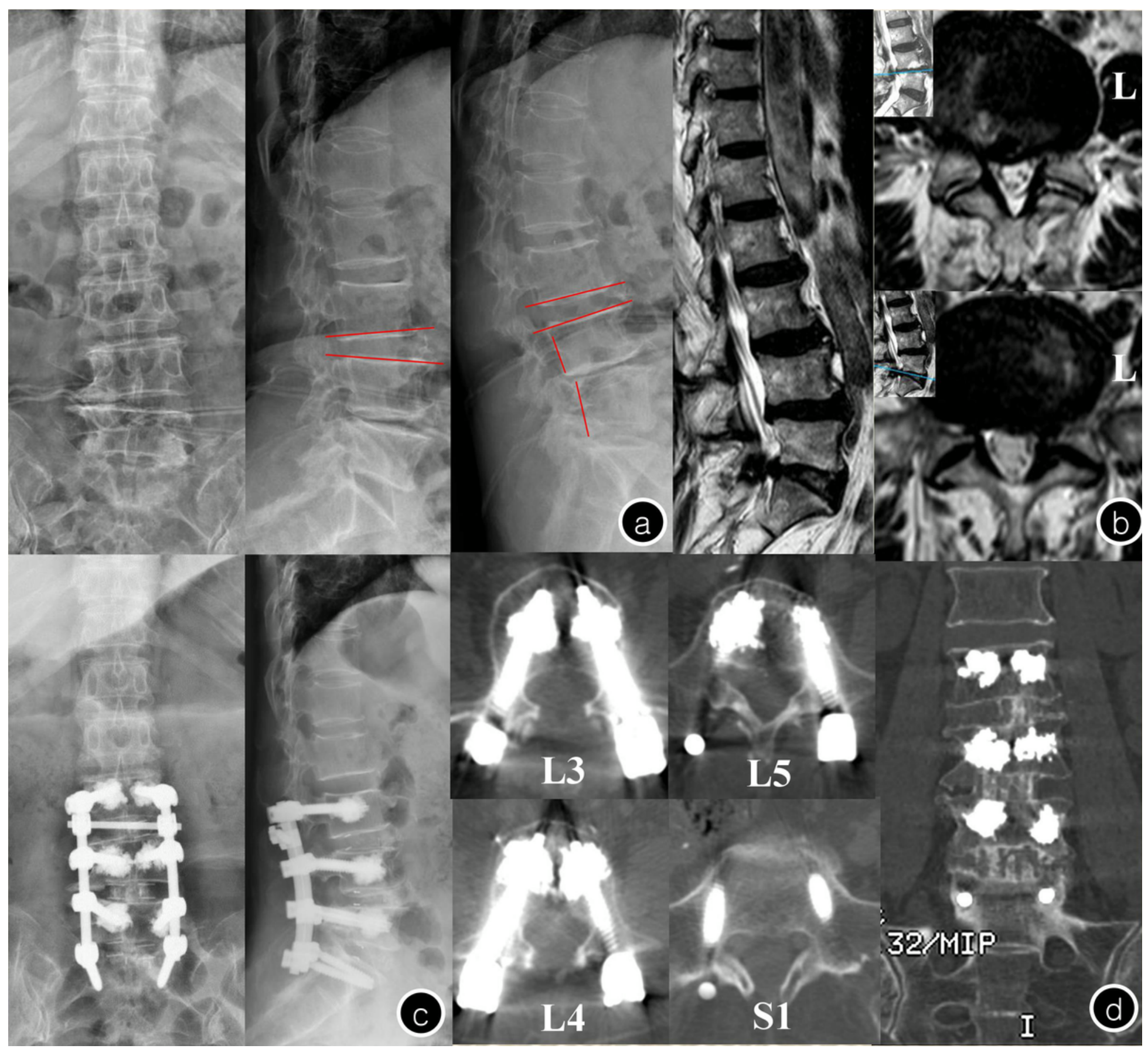

Figure 5

Female $₫ 75$-year-old $\otimes T=-3.8 \mathrm{SD}$ a. Preoperative $\mathrm{x}$-ray image showed lumbar vertebral degeneration with spinal instability in L3-L5; b. Preoperative MRI: L4/5 spinal canal stenosis and L5-S1 level lumbar disk herniation; c. Postoperative x-ray: the internal fixation was in good position and without serious leakage of cement; $d$. Five years after the operation, the CT showed that pedicle screw has not loosened and the L3-S1 was interbody fusion. 


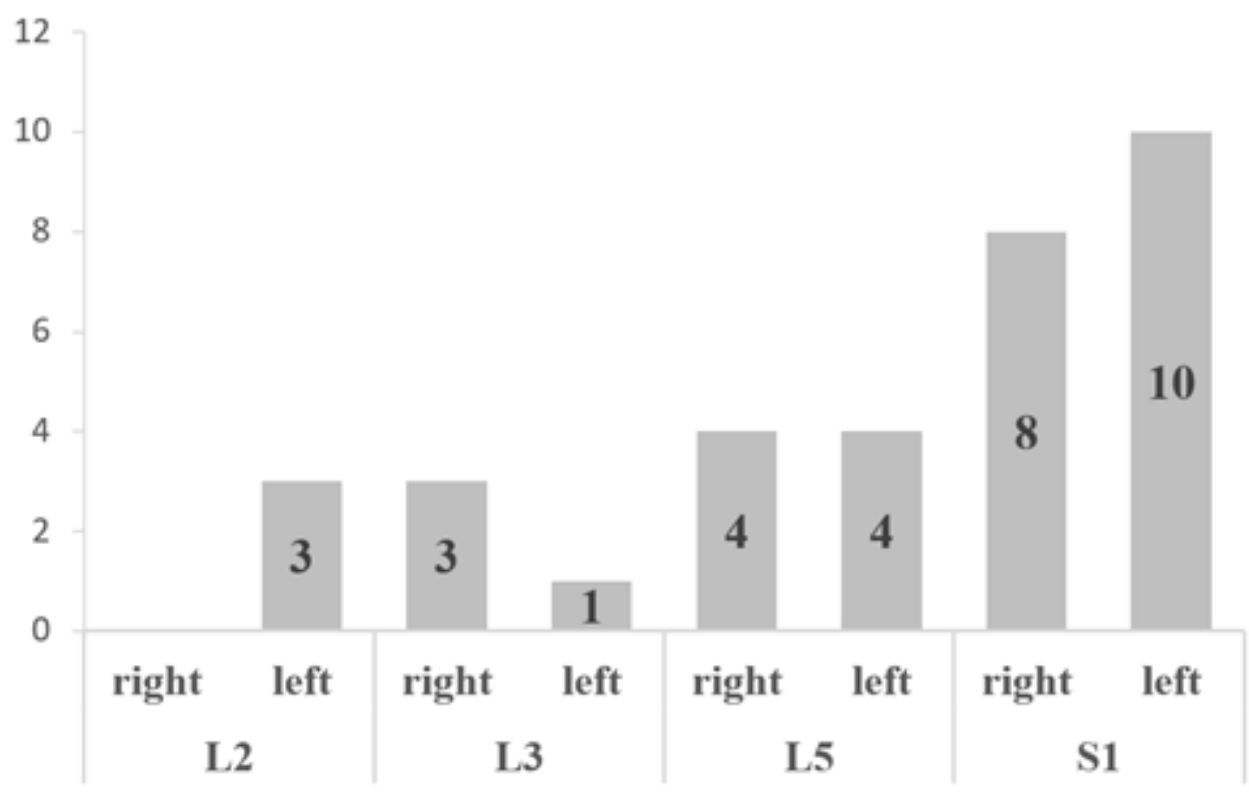

Figure 6

The distribution of screws loosening in the CPS group 


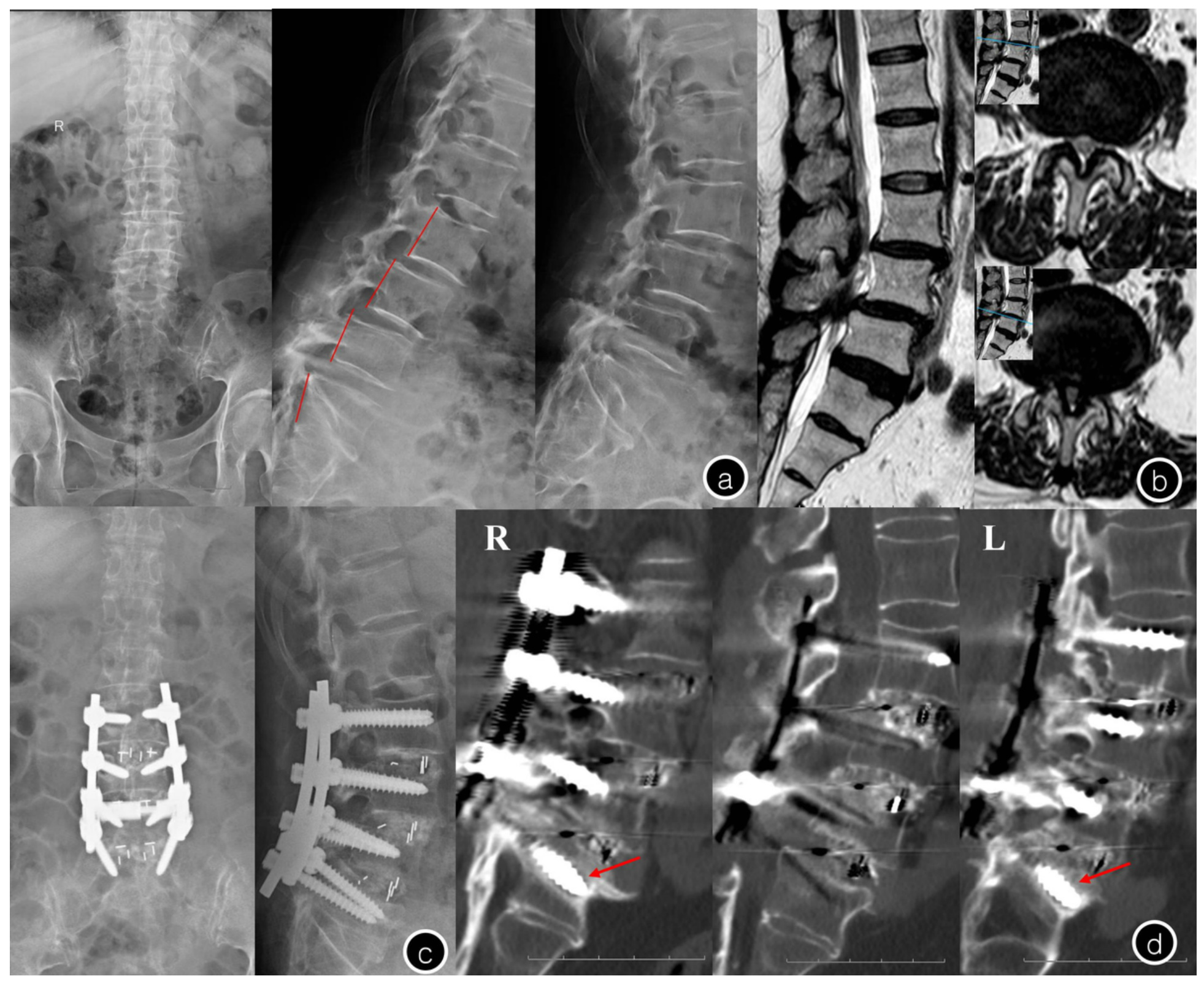

Figure 7

Female, 73-year-old, T= -2.5 SD a. Preoperative X-rays showed scoliosis in lumbar spine and L3-L5 spondylolisthesis; b. Preoperative MRI showed L3-5 level lumbar disk herniation with spinal canal stenosis; c. The lumbar anterior-posterior radiographs after surgery showed good reduction of spondylolisthesis; d. one year after the operation, CT illustrated halo sign appeared around S1 screws (arrows) and the S1 pedicle screw has loosened in bilateral. Meanwhile, L3-S1 was interbody fusion. 


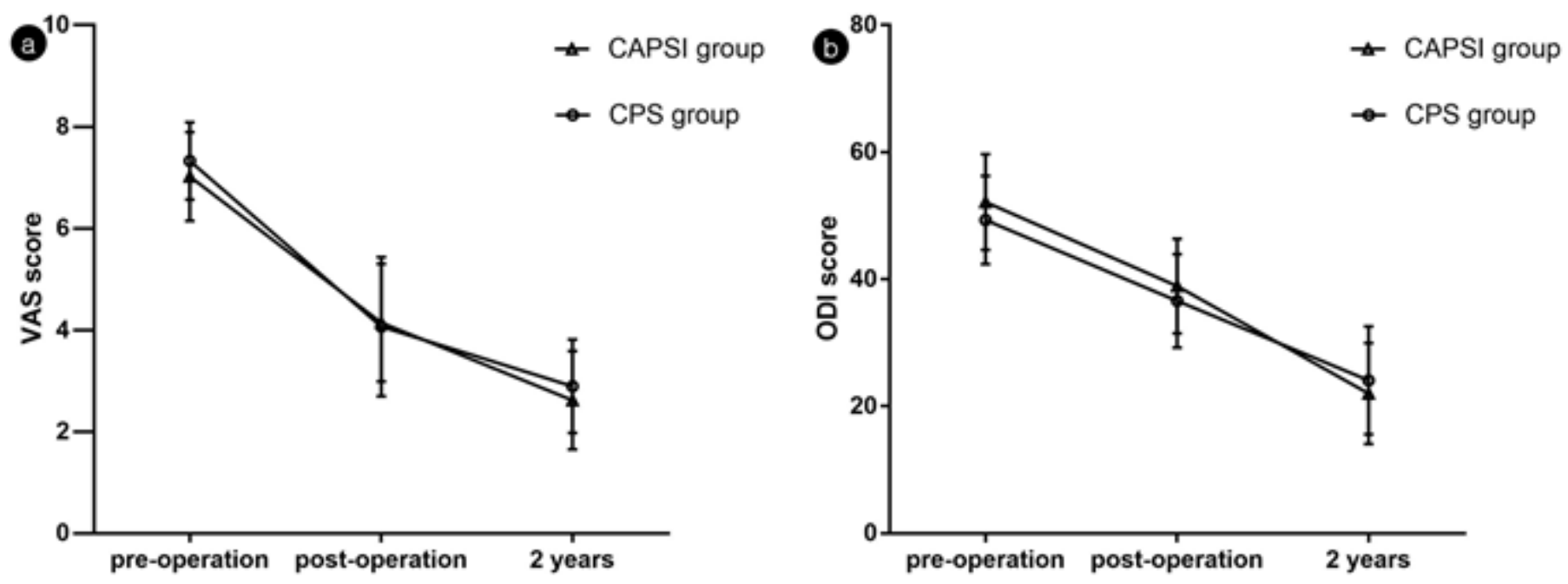

Figure 8

Changes in clinical results between the two groups during follow-up period, a. Mean Visual Analog Scale (VAS) score; b. Mean Oswestry Disability Index (ODI) score 